\title{
Relative Fluorescence Intensity Unit
}

National Cancer Institute

\section{Source}

National Cancer Institute. Relative Fluorescence Intensity Unit. NCI Thesaurus. Code C77535.

A unit of measurement that estimates the size or amount of material in a sample by normalizing its luminescence to that of a reference standard. 